\title{
Creación de réplicas de patrimonio escultórico mediante reconstrucción 3D e impresoras 3D de bajo coste para uso en entornos educativos
}

\author{
Creating replicas of scultural heritage through 3D \\ reconstruction and low cost 3D printer in Education
}

\author{
JoRGE DE LA TORRE-CANTERO \\ Universidad de La Laguna \\ jcantero@ull.edu.es \\ JÓSE L. SAORÍN \\ Universidad de La Laguna \\ jlsaorin@ull.edu.es \\ Cecile Meier \\ Universidad de La Laguna \\ alu0100305944@ull.edu.es \\ DÁmari MELIÁN-DíAz \\ Universidad de La Laguna \\ alu0100796810@ull.edu.es \\ Manuel Drago-Díaz alemán \\ Universidad de La Laguna \\ madradi@ull.es
}

Recibido: 10 de julio de 2014

Aprobado: 10 de marzo de 2015

\section{Resumen}

El proceso de elaboración de réplicas de patrimonio ha sido tradicionalmente desarrollado por organismos públicos, empresas o museos y no es de uso corriente en centros escolares. Actualmente existen tecnologías que permiten realizar réplicas de una manera muy económica. Los nuevos programas 3D basados en la reconstrucción a partir de fotografias y las impresoras $3 \mathrm{D}$ de bajo coste, permiten realizar maquetas del patrimonio con un importe muy inferior al tradicional.

En este artículo se describe el proceso de creación de una réplica de la escultura El Guerrero de Goslar del artista Henry Moore, situada en Santa Cruz de Tenerife. Para realizar esta reproducción, primero se han creado modelos 3D digitales utilizando las aplicaciones Recap 360, 123D Catch, Meshmixer y MakerWare. La réplica física, se ha reproducido en ácido poliláctico (PLA) mediante la impresora 3D MakerBot Replicator 2. Además, se incluye un análisis de costes utilizando, por un lado la impresora mencionada, y por otro, servicios de impresión 3D, tanto online como locales. Para finalizar, se ha realizado una acción puntual con 141 alumnos y 12 profesores de secundaria a los que se les ha pasado una encuesta de opinión sobre el uso de réplicas escultóricas en educación.

Palabras clave: impresión 3D, esculturas, patrimonio, educación.

De la Torre-Cantero, J., Saorín, J.L., Meier, C., Melián-Díaz, D., Drago-Díaz Alemán, M. (2015): Creación de réplicas de patrimonio escultórico mediante reconstrucción 3D e impresoras 3D de bajo coste para uso en entornos educativos. Arte, Individuo y Sociedad, 27(3) 429-446 


\begin{abstract}
The process of making replicas of heritage has traditionally been developed by public agencies, corporations and museums and is not commonly used in schools. Currently there are technologies that allow creating cheap replicas. The new $3 \mathrm{D}$ reconstruction software, based on photographs and low cost $3 \mathrm{D}$ printers allow to make replicas at a cost much lower than traditional.

This article describes the process of creating replicas of the sculpture Goslar Warrior of artist Henry Moore, located in Santa Cruz de Tenerife. To make this process, first, a digital model have been created using Autodesk Recap 360, Autodesk 123D Catch and Autodesk Meshmixer MarkerBot MakerWare applications. Physical replication, has been reproduced in polylactic acid (PLA) by MakerBot Replicator 2 3D printer. In addition, a cost analysis using, in one hand, the printer mentioned, and in the other hand, 3D printing services both online and local, is included. Finally, there has been a specific action with 141 students and 12 high school teachers, who filled a questionnary about the use of sculptural replicas in education.

Keywords: 3D printing, sculptures, heritage, education.
\end{abstract}

Sumario: 1. Introducción, 2. Antecedentes, 3. Fabricación de la réplicas de patrimonio escultórico, 3.1. Aspectos legales relacionados con las réplicas, 3.2. Fabricación de las réplicas de patrimonio escultórico, 4. Impresoras 3D en educación, 5. Creación de un modelo 3D digital, 5.1. Descripción de las tecnologías utilizadas, 6. Experiencia realizada, 6.1. Creación de la réplica, 6.2. Estimación del coste de la réplica utilizando servicios de impresión $3 \mathrm{D}, 6.3$. Divulgación del proceso de creación de réplicas en entornos educativos, 7. Conclusiones y futuros trabajos. Referencias.

Este artículo recoge resultados de la investigación «Mejora del Razonamiento Espacial y Visual mediante Herramientas Tecnológicas Avanzadas» (ESREVIC) financiada por Ministerio de Educación, Plan Nacional de I + D + I (2008-2011). Ref. TIN2010-21296-C02-02.

\title{
1. Introducción
}

La Conferencia General de la Organización de las Naciones Unidas para la Educación, la Ciencia y la Cultura de 1972 destaca la importancia de incrementar el respeto y el aprecio del patrimonio cultural mediante la educación (UNESCO, 2006). Según la Agencia Ejecutiva en el ámbito Educativo, Audiovisual y Cultural que analiza la Educación Artística y Cultural en el contexto escolar en Europa, un objetivo común en todos los países en la educación artística es la comprensión del patrimonio (Eurydice, 2009). El patrimonio cultural abarca monumentos como obras arquitectónicas, artísticas, arqueológicas, o conjuntos que tengan un valor universal excepcional desde el punto de vista de la historia, del arte o de la ciencia. El patrimonio escultórico es una parte importante del patrimonio artístico. Una estrategia utilizada habitualmente en la enseñanza del patrimonio escultórico es incluirlo en contenidos transversales dentro de diversas asignaturas o de manera directa en clases de historia, sociales, cultura o arte. Dentro de esta estrategia se incluyen visitas a museos, excursiones y utilización de distintos soportes gráficos. Las réplicas habitualmente se utilizan en los museos para reemplazar patrimonio cultural como obras escultóricas, arqueológicas $\mathrm{o}$ arquitectónicas que por su fragilidad, posible deterioro u otras razones, no pueden ser expuestas. Hasta ahora, para la elaboración de las réplicas se recurre a expertos de diferentes especialidades y se utilizan tecnologías y procedimientos complejos. Con la aparición de tecnologías de bajo coste es posible hacer extensible el uso de réplicas en entornos educativos. 


\section{Antecedentes}

Una réplica es una reproducción, con la máxima exactitud posible, del original de un objeto y puede ser de distinto material y tener diferente escala (Almagro Gorbea, 1988). Una de las razones por las cuales se realiza una réplica es para reemplazar una obra de gran valía y evitar que sufra deterioros. A las réplicas se les atribuye un valor destacado porque sirven para transmitir el arte al público en general, y sin su existencia sólo especialistas o investigadores tendrían acceso a obras de arte protegidas.

Un campo donde se usan réplicas desde hace más de cien años es la arqueología. Los restos arqueológicos muchas veces son delicados o están muy deteriorados y el uso de copias permite que el público en general pueda apreciarlos y acceda a los mismos sin el riesgo de dañarlos que conlleva (Crasbom Chavarría \& Orrego Corzo, 2007). Dentro de las investigaciones paleontológicas, el uso de réplicas se hace casi indispensable, ya que facilita de manera considerable el trabajo a los investigadores o museos (Arribas, et al., 2004). Las réplicas usadas en el campo del patrimonio arquitectónico se utilizan, recreando edificios históricos, en museos de miniaturas y en tiendas de coleccionismo. Otro caso, por ejemplo, en China se reproducen edificios o ciudades enteras a escala real. En 2012 se construyó una réplica de Hallstatt pueblo de Austria, declarado Patrimonio Mundial por la Unesco, en la provincia china de Guangdong (Morris, 2014). También está en construcción una réplica del centro sagrado de Tenochtitlán, la capital del Imperio Azteca, para la conservación del original (Hernández Martínez, 2007).

La copia del patrimonio artístico es muy frecuente para que los amantes del arte puedan adquirir una réplica exacta a un precio inferior al original. Las primeras copias conocidas de esculturas surgieron en el siglo II y I aC. Cuando los romanos realizaron réplicas de esculturas griegas clásicas hechas en el siglo $\mathrm{V}$ y IV aC. En la época del renacimiento los artistas comenzaron a usar réplicas de estatuas de la Antigüedad para el "dibujo de estatua", técnica que se extendió a finales del siglo XVII con el inicio de la Academias de Bellas Artes, por lo que se multiplicaron las réplicas en yeso, creando importantes colecciones en éstas escuelas. La creación de duplicados alcanzó un auge en el s. XIX cuando se crean varios museos de reproducciones. Durante el pasado siglo las réplicas de esculturas empezaron a perder aprecio, y se empieza a valorar el poder contemplar el original de la obra (Luzón Nogué, 2003). Un proyecto actual que trabaja con réplicas del patrimonio cultural y la creación de modelos digitales es el creado por MIDEA (Marcus Institute for Digital Education in the Arts), que trabaja entre otras cosas, en la realización de réplicas de patrimonio cultural para su preservación (Marcus Institute for Digital Education in the Arts, 2009).

Los proyectos con el uso de réplicas en museos o investigaciones han sido reservados para grandes empresas u organismos, pero han sido imposibles de introducir de manera generalizada en un entorno escolar. Los museos son las primeras instituciones que usan las réplicas con fines educativos, pero el acceso de alumnos a los museos es limitado. Sin embargo, con la aparición de nuevas tecnologías y la constante digitalización han aparecido nuevas iniciativas para conservar y divulgar el patrimonio.

El proyecto creado por distintos museos de Reino Unido junto al Departamento Geológico Británico (BGS), en el que se lanzó una base de datos con cientos de modelos de fósiles en tres dimensiones, disponibles para descargar, visualizar en formato digital 
e imprimir en 3D. Estos proyectos cuentan con un enfoque educativo y trabajan con centros escolares, organizando visitas guiadas o facilitando material y recursos. Además, este proyecto brinda la posibilidad de visualizar los fósiles en cualquier aula, en un ordenador con conexión a internet, además de poder imprimir una réplica si se dispone de una impresora 3D (British Geological Survey, 2013). No sólo en proyectos oficiales se puede encontrar patrimonio para uso personal o educativo. Existen galerías 3D de libre acceso y de descarga gratuita donde se encuentran réplicas digitales de patrimonio con la posibilidad de imprimirlas, realizando una reproducción física con cualquier impresora 3D. En éstas páginas web están disponibles para su descarga, por ejemplo, $E l$ David de Miguel Ángel (Fig. 1) o El pensador de Rodin (MakerBot, 2008). Debido a la aparición de cada vez más objetos digitalizados en $3 \mathrm{D}$, podemos pensar en la inclusión de modelos tridimensionales en la educación.

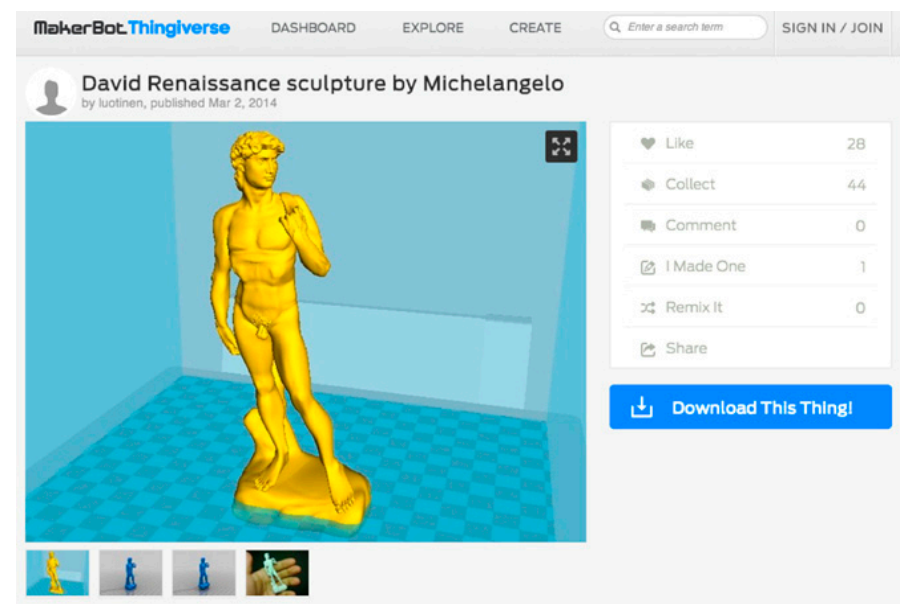

Figura 1. Modelo 3D de El David de Miguel Ángel, descargable en Thingiverse. (Imagen de la web: http://www.thingiverse.com/).

\section{Fabricación de réplicas de patrimonio escultórico}

\subsection{Aspectos legales relacionados con las réplicas}

La propiedad intelectual de una obra literaria, artística o científica corresponde al autor por el sólo hecho de su creación. Respecto a los materiales accesibles a través de la red, podemos leerlos, verlos u oírlos gratuitamente si así lo ha decidido su autor, sin embargo no podemos copiarlos, ni redistribuirlos sin su autorización. Al escanear una escultura con cualquier técnica, ya sea láser o fotografías, estamos realizando una copia de un objeto protegido por el Copyright. Por esta razón, copiar y distribuir una escultura requiere el permiso de la persona que tiene los derechos de reproducción (Weinberg, 2013).

Sin embargo, según consta en el apartado 2, del artículo 32 de la ley de propiedad intelectual (Boletín Oficial del Estado, 2006) "Cita e ilustración de la enseñanza: No necesitará autorización del autor el profesorado de la educación reglada para realizar actos de reproducción, distribución y comunicación pública de pequeños fragmentos 
de obras o de obras aisladas de carácter plástico o fotográfico figurativo, excluidos los libros de texto y los manuales universitarios, cuando tales actos se hagan únicamente para la ilustración de sus actividades educativas en las aulas, en la medida justificada por la finalidad no comercial perseguida, siempre que se trate de obras ya divulgadas y, salvo en los casos en que resulte imposible, se incluyan el nombre del autor y la fuente".

En este artículo, para ilustrar el proceso de creación de una réplica, se ha escogido la escultura El Guerrero de Goslar de Henry Moore (Castleford, Inglaterra, 1898-1986). Dicha obra está situada en la rambla de Santa Cruz de Tenerife (Canarias-España) y se colocó en 1977 para sustituir a Reclining Figure, del mismo artista, que había formado parte de la I Exposición Internacional de Esculturas en la Calle, celebrada en la ciudad en 1973. Forma parte del patrimonio de la ciudad y en 2007, el Gobierno de Canarias la ha declarado Bien de Interés Cultural (BIC) (Boletín Oficial de Canarias, 2007). Esta obra está realizada en bronce, con unas dimensiones de 170 x 360 x $181 \mathrm{~cm}$. y según el BOC de junio de 2007, es una "representación de un guerrero caído, figura recostada con la cabeza en un extremo y el escudo a los pies, en el lado opuesto" (Fig. 2). Curiosamente, la obra es una réplica del original que está en los jardines del Mönchehaus Museun für Moderne Kunst Goslar, en Alemania (Tourist Information Goslar, 2014). En 2008, el ayuntamiento de Santa Cruz cede la obra para la inuguración del Tenerife Espacio de las Artes (TEA) y es en este espacio cultural donde, en marzo de 2009, se celebra un taller infantil en el que los niños se introducían en el concepto de la escultura y sus peculiaridades acercándose a esta creación de Henry Moore, realizando reproduciones a escala de la obra original y transformándola mediante diferentes técnicas y procedimientos plásticos (La Laguna Ahora. El periódico digital de La Laguna, 2009). Finalmente, en mayo de 2009 vuelve a situarse en su ubicación original, en Las Ramblas de Santa Cruz, donde se encuentra actualmente. Las características comentadas hacen que sea una obra con gran potencial para la divulgación del patrimonio escultórico en entornos educativos. Debido a que Henry Moore murió en 1986, los derechos de copyright están vigentes; sin embargo, en este artículo sólo se pretende analizar la viabilidad técnica y económica de realizar réplicas con el objeto de utilizarlas con fines educativos.

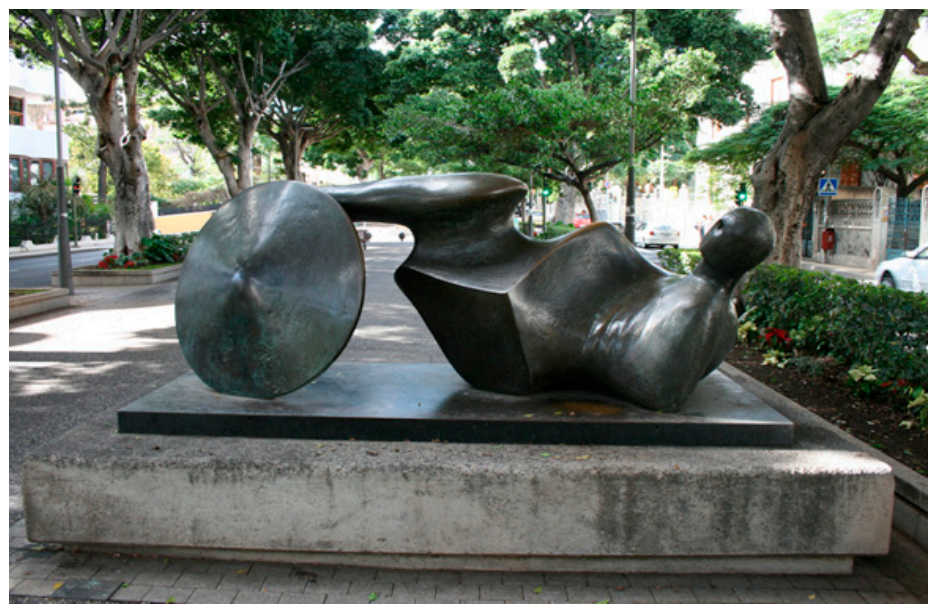

Figura 2. Escultura Guerrero de Goslar (Rambla de Santa Cruz de Tenerife). (Imagen de autoría propia). 


\subsection{Fabricación de réplicas de patrimonio escultórico}

Para crear una réplica de una escultura primero hay que realizar una copia del modelo actual que actuará de molde o referencia y luego materializar las réplicas propiamente dichas. Tradicionalmente, para crear la primera réplica se han utilizado varios procedimientos, entre ellos el sacado de puntos por coordenadas, o medidas, de la escultura. En este método, una vez se han obtenido los puntos se procede a realizar mediante técnicas manuales la réplica a escala. Otra manera de elaborar réplicas es el uso del pantógrafo. Con él, se pueden hacer copias tanto de paneles (relieves) como de figuras tridimensionales. El pantógrafo se utiliza sobre todo para tallas en madera y funciona como un fresado tridimensional (Educastur, 2008). Por otra parte, también se trabaja con moldes para elaborar una réplica. Sin embargo, un molde tiene durabilidad limitada y necesita de un proceso manual de limpieza de la figura. Las técnicas tradicionales de obtener réplicas representan una manera de trabajar costosa y lenta, además, en muchos casos no es posible el uso de moldes por la fragilidad del objeto que se ha de reproducir. Por ello, desde hace años se intenta mejorar este procedimiento mediante la incorporación de tecnologías digitales.

En una primera fase las tecnologías aplicadas procedían del mundo de la topografía y estaban basadas en aplicaciones orientadas a la creación de modelos digitales del terreno (MDT). Existen diferentes técnicas topográficas para generar los puntos de la malla 3D del territorio; puede hacerse mediante aparatos tradicionales de topografía, mediante fotografías (fotogrametría) o mediante escáner láser (Gonizzi Barsanti, Remondino, \& Visintini, 2012). El uso de estas nuevas tecnologías aplicadas al patrimonio, simplifica mucho el proceso de creación de réplicas, aunque el coste siempre ha sido muy alto. $\mathrm{Al}$ mismo tiempo hay que tener en cuenta que se necesita una formación adecuada para poder llevar a cabo las copias con éstos métodos, y además se requieren tecnologías poco accesibles (Lerma, Cabrelles, \& Seguí, 2011).

Una vez terminado el proceso de digitalización 3D del objeto, se realiza la reproducción física de la réplica. Una de las tecnologías que posibilitan la fabricación de las réplicas es la impresión $3 \mathrm{D}$, sin embargo uno de los grandes factores que ha limitado el uso de esta tecnología en las aulas ha sido el precio. Hasta el año 2005, una máquina de impresión 3D tenía un precio superior a 20.000 dólares, por lo que sólo los grandes centros se la podían permitir (Johnson, Coates, Hager, \& Stevens, 2009).

En el año 2001, en el Media Lab del MIT se crea el proyecto FabLab en colaboración con el Centre for Bits and Atoms (CBA) con el objeto de difundir las tecnologías de fabricación digital. Desde entonces el movimiento fablab se ha globalizado, ayudando a popularizar las tecnologías de fabricación 3D de bajo coste. (Troxler \& Wolf, Bending the Rules. The Fab Lab Innovation Ecology, 2010). En 2005, surge el proyecto RepRap en la Universidad de Bath (Reino Unido) con el objetivo de abaratar los costes de la impresión 3D. Esta iniciativa dio lugar a la popularización de dichas máquinas, ya que los precios de dichas impresoras se redujeron hasta estar en torno a los 1000 dólares. Uno de los fundadores del proyecto RepRap, Zach Smith, creó en 2009 la empresa MakerBot cuyo objetivo era vender kits de impresoras 3D para que todo el mundo pudiera construirse una, por menos de 1000 dólares. A partir de ese momento, las impresoras de bajo coste se han popularizado y ha surgido una industria alrededor de ellas. La aparición de esta nueva gama de impresoras permite a los centros educativos 
disponer de ellas y utilizarlas en su docencia. Por lo tanto, una vez superada la barrera del precio, es necesario disponer de metodologías y recursos docentes que nos permitan sacar partido de esta tecnología en nuestros entornos educativos (Canessa, Fonda, \& Zennaro, 2013).

\section{Impresoras 3D en la educación}

Dentro de los distintos informes internacionales sobre educación existe uno específico en relación a las tecnologías, que se ha convertido en un referente: el "Informe Horizon". En España, este informe se presenta desde el Instituto de Tecnologías Educativas (ITE), dependiente del Ministerio de Educación y responsable de la integración de las TIC en la enseñanza (TICE). Según el informe Horizon una de las tecnologías que se prevé que tenga impacto en la educación es la impresión 3D (New Media Consortium, 2013).

La impresión $3 \mathrm{D}$ existe desde los años 70 , sin embargo no se introdujo en el ámbito educativo hasta el año 2000 y fue a través de centros de educación superior. Una de las pioneras fue la Universidad de Illinois, en 2002, donde se utilizó la tecnología de prototipado rápido para realizar un estudio experimental sobre la mejora de las capacidades espaciales de los estudiantes (Czapka, 2002). En 2009, en la Universidad del Estado de Georgia se introdujo un módulo sobre la tecnología de prototipado rápido dentro de la asignatura Ingeniería Gráfica (Wayne M. Johnson, 2009). En 2012, en la Universidad de Alabama y la Universidad de Nevada incorporaron la impresión 3D como un servicio más dentro de las bibliotecas, con el objetivo de atraer nuevos usuarios (Scalfani \& Sahib, 2013).

En entornos preuniversitarios, destaca el proyecto KIDE iniciado por Dejan Mitrovic en Londres en el año 2009. Fue diseñado para los niños de las escuelas primarias y desde entonces ha realizado su actividad en varios países de todo el mundo. KIDE es un programa educativo que combina juegos creativos y talleres para desarrollar habilidades constructivas y de ingeniería de diseño en los niños a través del juego, y les permite proyectar y fabricar sus propios juguetes $3 \mathrm{D}$ en las escuelas. Se trata de un sistema completo, que incluye impresoras 3D con software sencillo y muchos juegos con tareas creativas (Fig. 3). En el Reino Unido, el gobierno ha apostado, de forma clara, por esta tecnología. El Departamento de Educación de este país, ha incluido este tipo de tecnologías en el currículum, para que los alumnos las conozcan desde muy jóvenes (Department for Education of United Kingdom, 2013). 


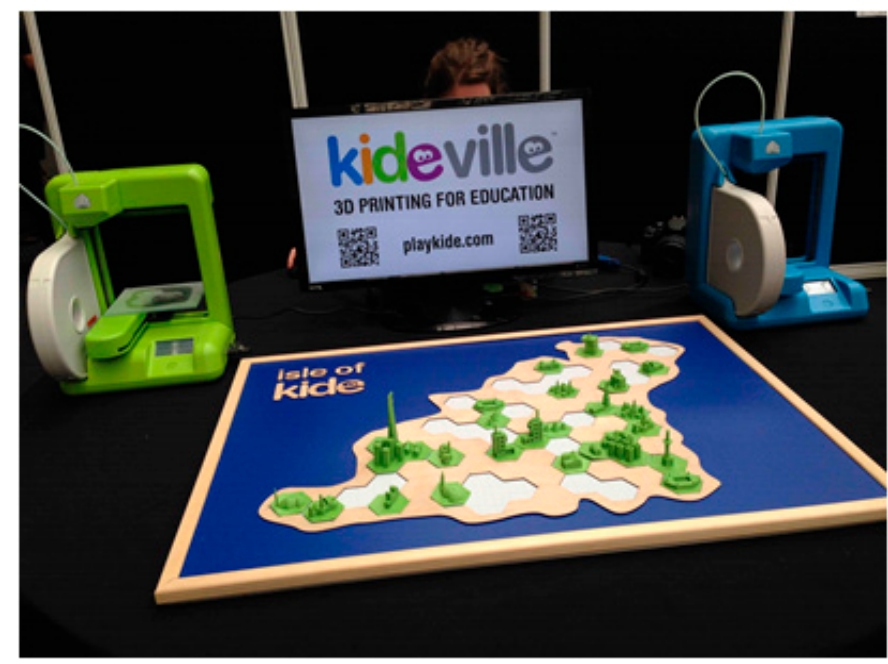

Figura 3. Proyecto KIDE. 3D Printshow, Londres 2013. (Imagen de autoría propia).

En España existen pocas referencias bibliográficas sobre la inclusión de estas tecnologías en ámbitos educativos. En Tenerife se han realizado algunas experiencias, como por ejemplo el Proyecto de Innovación Docente de la Universidad de la Laguna (Fig. 4) denominado "Transformación de diseños virtuales 3D en maquetas reales mediante el uso de impresoras 3D de bajo coste" (Saorín, de la Torre Cantero, Zanardi Maffiote, Martín Dorta, \& Carbonell Carrera, 2013). También en esta misma isla, surge una interesante iniciativa de divulgación de estas tecnologías en centros escolares de secundaria, promovida desde el Cabildo de Tenerife denominada MoveFab (MoveFab, 2013). Como resultado de dicha divulgación se han diseñado una serie de talleres para promover su utilización en centros educativos (Martín Dorta, de la Torre Cantero, \& Saorín Pérez, 2014).

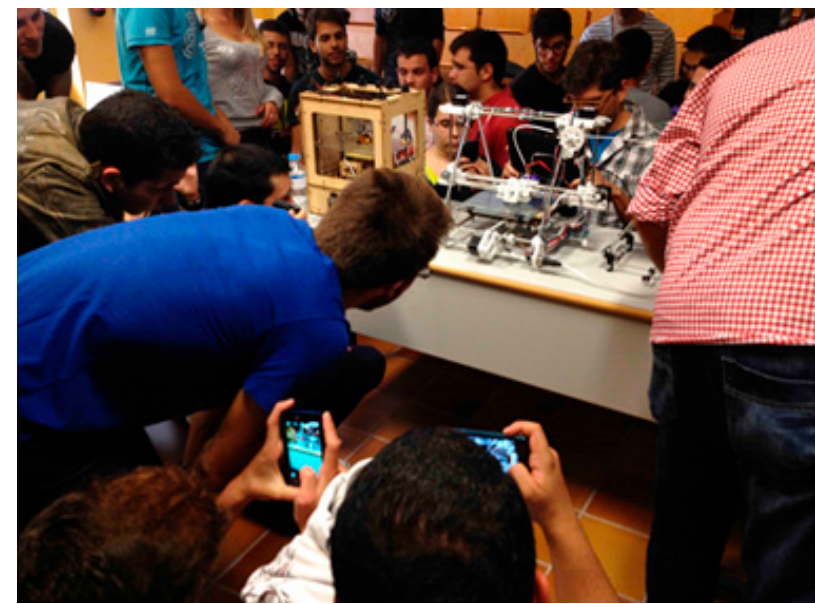

Figura 4. Proyecto de innovación docente en la Universidad de La Laguna, 2013.

(Imagen de autoría propia). 


\section{Creación de un modelo 3D digital}

A pesar de que las tecnologías de impresión 3D han bajado de precio, para poder utilizarlas es necesario disponer de modelos 3D digitales; y aunque existen repositorios de modelos 3D, si queremos imprimir prototipos propios es necesario ser capaz de generarlos y editarlos. Los modelos 3D pueden realizarse de objetos de diseño personal o bien de elementos ya existentes, como puede ser el patrimonio escultórico de una ciudad. En este segundo caso, una técnica para obtener un modelo 3D es la creación de objetos tridimensionales a partir de fotografías. Esta técnica en continuo desarrollo, es una alternativa al escáner láser, pero más económica (Torres, Arroyo, Romo, \& De Haro, 2012). Existen varios programas con este tipo de funcionamiento (Photomodeller, Bundler, PMVS2, VisualSFM, Insight3D, AgiSoft PhotoScan, Pix4D, Microsoft Photosynth, 123D Catch, ReCap 360, etc.). De manera generalizada se dividen en tres grupos. Unos que trabajan con algoritmos individuales y resuelvan tareas específicas dentro del proceso de reconstrucción. Otros programas que crean un modelo 3D a partir de fotografías y por último programas online, donde se envían las imágenes por internet, se procesan de forma remota en "la nube" y de este modo se obtiene el modelo 3D. Algunos de estos programas son costosos y necesitan formación específica, sin embargo están apareciendo alternativas asequibles y de manejo sencillo. Para la realización del proceso que se describe en este trabajo se han utilizado dos programas gratuitos, diseñados para su uso en entornos educativos, que además son de manejo fácil e intuitivo y que realizan la labor de reconstrucción 3D en la nube: Autodesk 123D catch y Autodesk Recap 360.

A pesar de que están diseñados para su aplicación en contextos educativos, estos programas tienen grandes prestaciones que permiten utilizarlos, además, en la generación de modelos 3D en proyectos relevantes. Por ejemplo, Tomas P. Kersten demuestra su uso gratuito, inmediato y efectivo para la creación 3D de objetos arqueológicos (Kersten \& Lindstaedt, 2012). Otros autores, comparan varios programas de reconstrucción automática y destacan Autodesk 123D Catch por su ajustado precio, la facilidad de uso y las posibilidades de edición del modelo (Brutto \& Meli, 2012). Autodesk 123D Catch también se emplea para la reconstrucción de patrimonio arquitectónico con excelentes resultados (Manferdini \& Galassi, 2013). Otros autores demuestran, igualmente, el uso efectivo de este programa para obtener modelos que pueden ser la base para extraer información geométrica precisa (Tsioukas, 2013).

En el mismo sentido, también el programa Autodesk Recap 360 se está usando con fines profesionales. El Museo del Mediterráneo y de Oriente Próximo Antigüedades (Medelhavsmuseet) en Estocolmo se sirve, entre otros programas, del Recap 360 para que los visitantes puedan interactuar y rotar modelos 3D de una colección de seis momias (Swedish ICT, 2014).

Una vez obtenido un modelo tridimensional de malla, generalmente hay que editarlo antes de proceder a su impresión. Al igual que en los programas de generación de modelos 3D a partir de fotografías, existen muchos programas (Blender, 3DStudio, ZBrush, MudBox, Maya, Meshmixer...) que permiten editar dichos modelos. En algunos proyectos de documentación de patrimonio (Pomaska, 2013) se están utilizando estas tecnologías. 


\subsection{Descripción de las tecnologías utilizadas}

Autodesk 123D Catch es un programa gratuito que permite generar modelos 3D a partir de la captura fotográfica de un objeto. Los procesos de cálculo se realizan por computación remota en la nube. Autodesk 123D Catch aparece en noviembre de 2011 en su versión para PC, en mayo de 2012 se crea la versión para iPad y en septiembre del mismo año, surge la versión para iPhone.

Al igual que 123D Catch, ReCap 360 también pertenece a la familia de Autodesk y reproduce en tres dimensiones objetos o lugares a los que previamente se les ha realizado una serie de fotografías. Este programa sólo existe en su versión online, y no necesita descargar ningún programa, ni precisa instalación en el equipo.

El programa 123D Catch dispone de su propio editor de mallas 3D online. Después de subir las fotografías, el programa nos devuelve una malla 3D que podremos modificar los modelos con seis sencillas herramientas. Éstas sirven principalmente para recortar el entorno del objeto fotografiado y cerrar agujeros que se hayan podido generar. En cambio, Autodesk ReCap 360 no dispone de un editor, por lo que es necesario utilizar otros programas para producir el modelo obtenido. Para el trabajo descrito en este artículo se ha utilizado la aplicación gratuita Autodesk Meshmixer.

Meshmixer es una herramienta para mezclar, esculpir, pintar, limpiar y reparar grandes mallas 3D. El objetivo de este programa es facilitar el proceso a aquellos que no son profesionales del modelado 3D. Es un medio simplificado para editar mallas de modelos tridimensionales (Schmidt \& Ratto, 2013).

Una vez se ha terminado de editar la malla, para poder ser fabricado el modelo 3D con una impresora 3D, se exporta a un formato estándar (STL, OBJ). Después se ha de generar el fichero (G-code) que sea reconocido por la impresora 3D. Para realizar esta transformación, se ha utilizado el programa gratuito MakerWare, que incorpora todas las opciones necesarias para visualizar, rotar, escalar y mover los diseños que pueden ser utilizados en las impresoras de la empresa MakerBot, entre ellas la Replicator 2 utilizadas en este trabajo. MakerBot Replicator 2 tiene un coste aproximado de 2.500 euros e imprime modelos con medidas de hasta 240 x 140 x $150 \mathrm{~mm}$. MakerBot Replicator 2 trabaja con PLA (polylactic acid), un termoplástico biodegradable, ligero y fácil de utilizar para impresión 3D. Su punto de fusión es más bajo que el plástico ABS (Acrylonitrile Butadiene Styrene) utilizado en el mismo tipo de impresoras 3D y por lo tanto, permite una impresión más estable.

\section{Experiencia realizada}

En esta experiencia se trata de ver la viabilidad técnica y económica de obtener un modelo 3D físico completo a escala de una escultura ubicada en la calle. Este proceso se ha realizado en otras investigaciones (Bourke, 2012), aunque sin la posibilidad de conseguir un modelo tridimensional impreso, debido al elevado importe que hasta hace poco suponía el uso de una impresora 3D. Para el proceso se elige una de las esculturas más importantes de Santa Cruz de Tenerife. Se trata de El Guerrero de Goslar de Henry Moore (Fig. 2), declarada como Bien de Interés Cultural por el Gobierno de Canarias en 2007. Una vez realizada la réplica, se presenta el trabajo a alumnos y profesores 
para disponer de una valoración sobre la posibilidad de incluir este recurso en entornos educativos.

\subsection{Creación de la réplica}

A la hora de elegir una escultura para su reconstrucción 3D digital a base de fotografías y posterior impresión, se ha tenido en cuenta que la obra se encuentra en un lugar de fácil acceso, debido a su ubicación en medio de una rambla peatonal; por lo tanto, se puede rodear por completo sin peligro, ni dificultad y realizar las fotografías necesarias para su reconstrucción 3D digital. Además, posee unas medidas de 170 x 360 x $181 \mathrm{~cm}$., facilitando, de esta manera, la posibilidad de visualizarla desde todos los ángulos y también desde un punto de vista cenital. Una parte importante del proceso es la iluminación de la escultura, procurando disponer de una luz homogénea y evitar una luz muy lateral, o tomar las fotografías a contraluz. Asimismo, es preciso encuadrar la escultura por completo en cada fotografía y enfocar correctamente.

Se realizaron 59 fotografías desde todos los ángulos de la escultura. Para este proceso se rodeó la escultura 4 veces. Tras completar la primera vuelta de fotografías desde un punto de vista de contrapicado, se comenzó la segunda vuelta desde un punto de vista medio y así hasta sacar las fotografías desde arriba, lo más cenital posible. Entre fotografía y fotografía se procura mantener aproximadamente la misma distancia hasta la escultura.

Para tomar las fotografías se utilizó una cámara Canon 400D. Una vez se obtenidas las imágenes necesarias, se exportan dichas fotografias a un ordenador. Como se ha comentado, se va a realizar la reconstrucción 3D con dos programas (ReCap360 y $123 \mathrm{D}$ Catch) que procesan la información fotográfica en la nube. Esta manera de trabajar posibilita obtener un modelo $3 \mathrm{D}$ sin necesidad de poseer un ordenador de alta gama. Esto es debido a que en la computación remota, los cálculos complejos, necesarios para la construcción digital de un modelo tridimensional, se realiza en servidores especializados para estas tareas. El trabajo de reconstrucción, puede ser lento (alrededor de una hora) ya que hay que enviar las fotos al servidor, procesar la información y devolver el resultado en forma de un fichero 3D. Una vez finaliza el proceso, el programa genera un aviso automático.

Como resultado, se obtiene un modelo 3D digital que se puede exportar en los formatos (obj, stl...) que son admitidos por otros programas de modelado 3D. Debido a que se parte de fotos realizadas en la calle, se restituye también el entorno alrededor de la escultura (Fig. 5) por lo que será necesario un procesamiento posterior del mismo. En la versión para ordenador de 123D Catch podemos realizar un primer postprocesado del resultado sin necesidad de usar, instalar o aprender otros programas. En este caso, sin embargo los modelos obtenidos de los dos programas serán editados en un programa diferente, aunque igualmente gratuito, Meshmixer. 


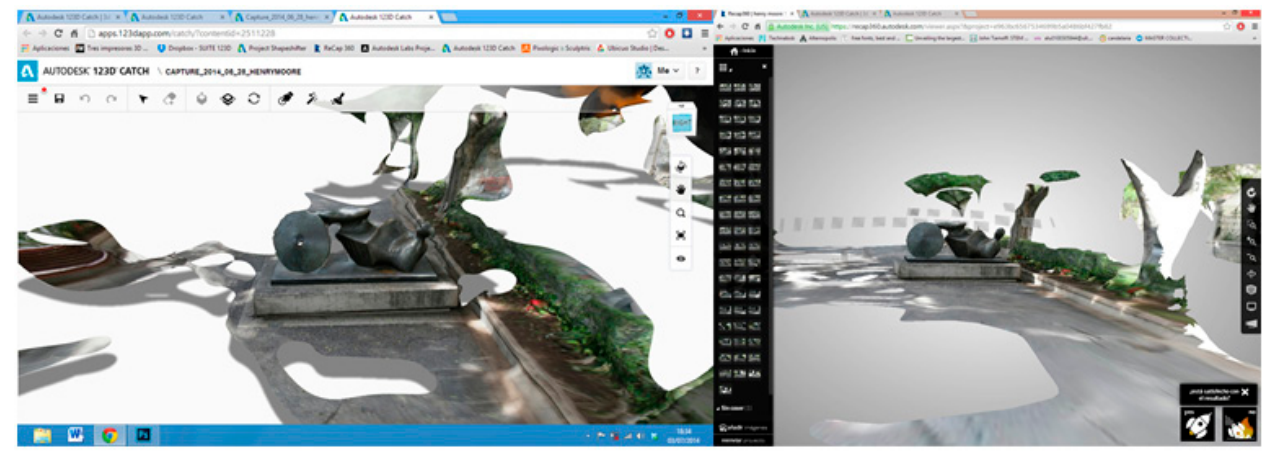

Figura 5. Restitución 3D con 123D Catch (izq.) y con Recap 360 (dcha.).

(Imagen de autoría propia).

Para comenzar a trabajar, se importa el archivo que queremos editar. Nos encontramos con varias herramientas para mejorar el modelo importado. Meshmixer posibilita exportar el modelo tridimensional digital en varios formatos. Para este ejemplo se ha utilizado ".obj", que es un formato admitido por MakerWare, el programa de manejo de la impresora 3D. En la figura 6 se puede ver los resultados obtenidos después de editar la figura. Se observa que el programa Recap360 proporciona una construcción tridimensional más parecida al original, con más detalles.

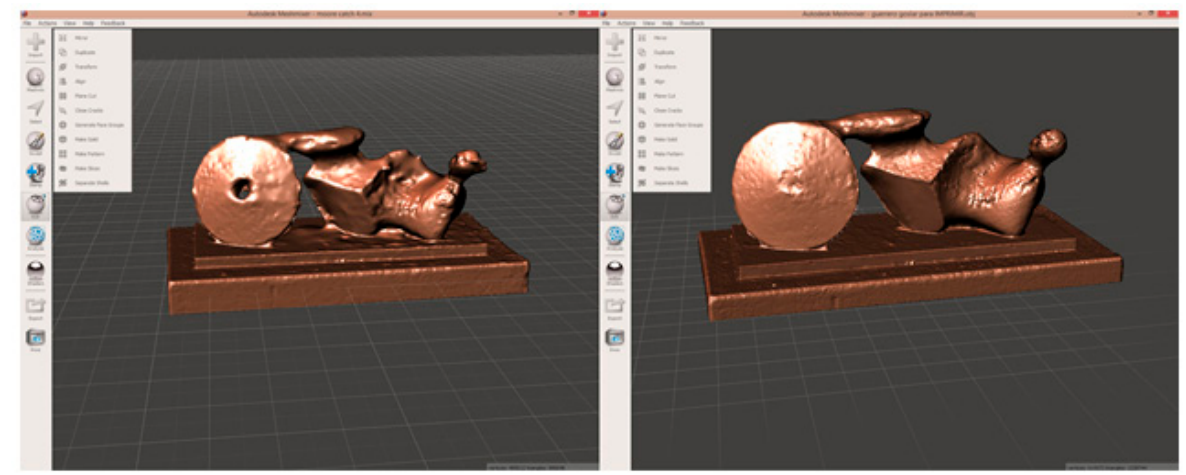

Figura 6. Modelos 3D generado en 123D Catch (izq.) y ReCap 360 (dcha.) editados en Meshmixer. (Imagen de autoría propia).

Una vez disponemos del fichero en formato ".stl" o ".obj" de El Guerrero de Goslar se imprime mediante una MakerBot Replicator 2 una réplica en PLA (polylactic acid) (Fig. 7). Para poder imprimir en una de estas impresoras 3D primero hay que generar, mediante el programa MakerWare suministrado por la impresora, un fichero G-code de extensión X3G que será el que se envíe a la impresora. Para este trabajo se ha realizado una réplica de $5,3 \times 10 \times 3,9 \mathrm{~cm}$. y 22 gramos de peso. Dicha réplica, con los ajustes de impresión seleccionados ( $10 \%$ relleno, grosor de capa $0,2 \mathrm{~mm}$.) ha necesitado aproximadamente 1,5 horas para estar finalizada. El coste de nuestra replica, teniendo en cuenta que $1 \mathrm{~kg}$. de PLA cuesta alrededor de 40 euros, será por lo tanto, aproximadamente de 0,9 Euros (sin incluir la amortización de la impresora 3D). 


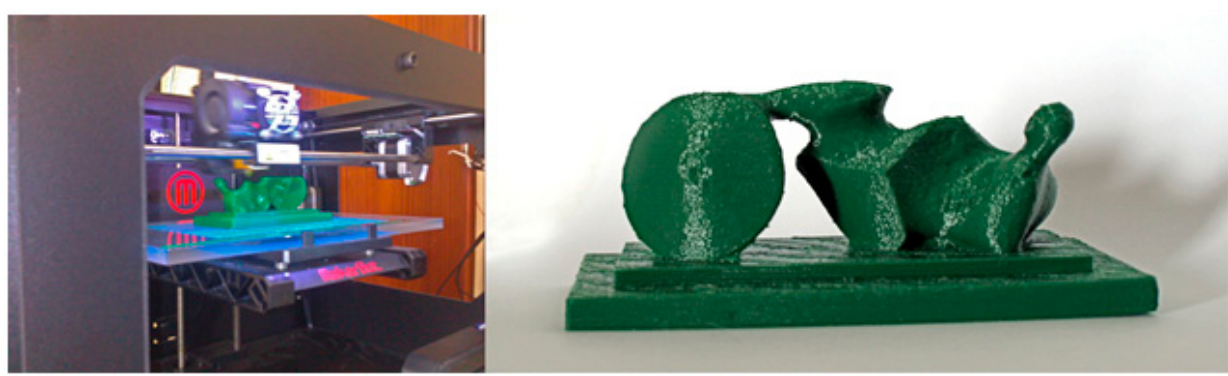

Figura 7. Impresora MakerBot Replicator 2 imprimiendo réplica del Guerrero de Goslar en PLA. (Imagen de autoría propia).

\subsection{Estimación del coste de la réplica utilizando servicios de impresión $3 D$}

Si no se dispone de una impresora 3D existe la alternativa de utilizar servicios de impresión online o servicios locales de impresión. La gran ventaja de poseer una impresora 3D, es que después del gasto inicial se puede imprimir siempre que se quiera a un coste muy bajo, ya que el precio del material es mínimo y se gasta poco. El problema es que la mayoría de impresoras asequibles en entornos escolares sólo permiten imprimir en plástico termofusible (PLA, ABS...). Cuando se necesita calidad, volúmenes mayores o materiales diferentes hay que acudir a un servicio especializado. Por eso, junto a la comercialización de las impresoras 3D, han proliferado los servicios de impresión 3D; tiendas que poseen varias impresoras desde las más básicas hasta máquinas profesionales y ofertan el servicio de impresión bajo pedido. Estos servicios de impresión externos pueden salir mucho más baratos si sólo se requiere un número limitado de piezas y en materiales específicos. Se podrán hacer reproducciones en diversos formatos, colores o materiales, aunque el precio varía bastante según el material o tamaño. Para este artículo se han estudiado tres servicios de impresión que funcionan de manera online (Sculpeo, Shapeways, e i.materialise) y uno de impresión local ubicado en Tenerife (Manzanas y 3D).

Shapeways es uno de los primeros servicios de impresión 3D que apareció en Internet. Fue fundada en 2007 en Holanda, y en la actualidad cuenta con sedes en Nueva York, Holanda y Seattle. Dispone de una librería online que cuenta con decenas de miles de objetos diseñados por usuarios y gran variedad de materiales, desde termoplásticos, plata, cerámica o piedra arenisca. Otra de las empresas con servicio de impresión 3D, es Sculpeo, con centros en California y Francia, que cuenta con un buen sistema de envío online de ficheros en 3D y entrega del modelo impreso en diferentes materiales. Asimismo dispone de una librería de piezas con aproximadamente 30.000 objetos creados por usuarios. Y finalmente otro servicio de impresión online analizado para ese artículo es i.materialise. Creada en 1990, esta empresa tiene características similares a las dos empresas anteriores.

En el caso de utilizar servicios online, el precio de imprimir una réplica del mismo tamaño de la realizada en la impresora MakerBot descrita anteriormente (5,3 x 10 x 3,9 $\mathrm{cm}$.), dependerá del material elegido para realizar dicha réplica. Existen multitud de materiales disponibles en cada una de las plataformas, pero a efectos de comparación, en la tabla 1 se pueden ver las valoraciones de distintas opciones. Cada uno de los servicios 
ofrece calidades que pueden ser diferentes entre sí, por eso los precios sólo nos dan una idea aproximada del coste comparado. Existe la posibilidad de imprimir en materiales como acero inoxidable, bronce, plata, ... pero las tarifas suben considerablemente. En la tabla 1 se ha incluido el coste de la réplica en acero inoxidable y bronce para tener una idea de la magnitud de los precios.

\begin{tabular}{|c|c|c|c|}
\hline Material & Sculpeo & i.materialise & Shapeway \\
\hline Material Plástico & $64,31 €$ & $90,03 €$ & $39,22 €$ \\
\hline Resina & $147,72 €$ & $101,50 €$ & $120,41 €$ \\
\hline Cerámica & $30,13 €$ & $22,28 €$ & $18,32 €$ \\
\hline Acero inoxidable & --- & $218,22 €$ & $321,65 €$ \\
\hline Bronce & --- & --- & $641,82 €$ \\
\hline
\end{tabular}

Tabla 1. Coste de la réplica en distintos materiales y servicios de impresión 3D online.

Del mismo modo se pueden utilizar tiendas de impresión locales y ahorrar los gastos de envío. En nuestro caso, en Santa Cruz de Tenerife existe, desde finales del año 2013, una tienda que presta este servicio (Manzana y 3D). Cuenta con varias impresoras entre ellas algunas de bajo coste como la Replicator y otras de gama más alta como la Objet para poder ofrecer a sus clientes variedad de calidades y precios. El importe de la creación de un modelo en la impresora MakerBot Replicator 2 se calcula según peso. En este caso el peso del modelo es de 22 gr. que multiplicado por $1.05 € / g r$., su precio ascendería a unos $23,10 €$.

\subsection{Divulgación del proceso en entornos educativos}

Para valorar las posibilidades del uso de réplicas con fines educativos, se realiza dentro del proyecto MoveFab, una acción de divulgación del proceso descrito en este artículo a varios centros escolares. Dicha presentación se realizó en la Casa de la Cultura del municipio de los Realejos (Tenerife) en junio de 2014. Además de la presentación, los participantes realizaron un taller donde utilizaban 123D Catch para realizar un proceso similar al descrito en el artículo. Participaron un total de 141 alumnos, divididos en 10 grupos de niveles educativos comprendidos entre $1^{\circ}$ y $4^{\circ}$ de la ESO, así como 12 profesores. Todo este colectivo tuvo la oportunidad, no sólo de ver el proceso de creación de réplicas, sino también de apreciar distintos tipos de réplicas de patrimonio (arqueológico, escultórico, natural...). Entre ellas, los participantes tuvieron en sus manos, la reproducción de la obra de Henry Moore, El Guerrero de Goslar, descrita en este artículo (Fig. 8). 


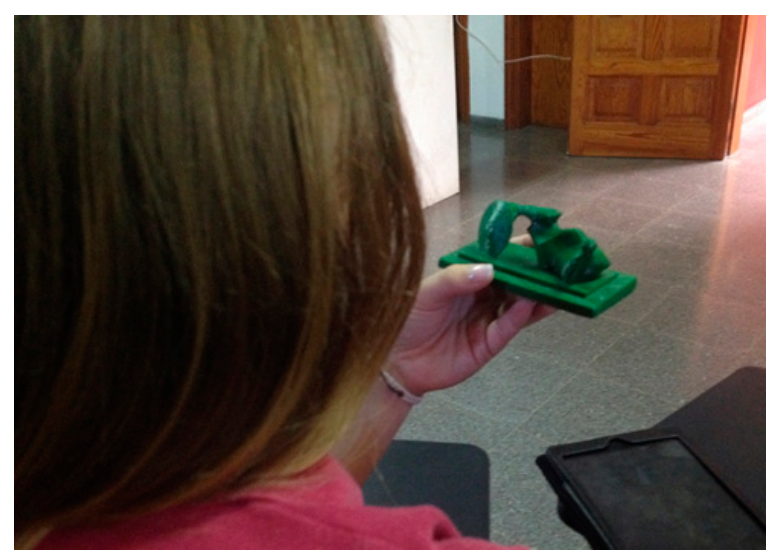

Figura 8. Alumna manipulando una réplica de la escultura El Guerrero de Goslar. (Imagen de autoría propia).

Una vez terminada la presentación y el taller, los profesores y los alumnos rellenaron una encuesta sobre el uso de réplicas en educación. Las preguntas se puntuaban de acuerdo a una escala Likert, de 1 a 5, donde 5 era totalmente de acuerdo y 1 totalmente en desacuerdo. Los resultados de la misma pueden verse en la tabla 2. Es importante señalar que esta encuesta se ha realizado para tener una primera valoración de lo que piensan alumnos y profesores sobre el uso y la creación de réplicas en educación. No se pretende con ello obtener resultados concluyentes, sino tener una idea inicial de lo que opinan los posibles usuarios del proceso descrito en este artículo. Cabe destacar que el $80 \%$ de los alumnos y profesores nunca habían visto funcionar en directo una impresora $3 \mathrm{D}$.

\begin{tabular}{|l|c|c|}
\hline \multicolumn{1}{|c|}{ Cuestión } & Profesores & Alumnos \\
\hline $\begin{array}{l}\text { Las réplicas 3D de obras escultóricas son un buen complemento } \\
\text { para la formación sobre el patrimonio }\end{array}$ & 4,25 & 4,01 \\
\hline $\begin{array}{l}\text { Me gustaría tener un repertorio de réplicas de patrimonio escultórico } \\
\text { para el aprendizaje y la enseñanza en el aula }\end{array}$ & 4,00 & 3,74 \\
\hline Me gustaría saber cómo generar réplicas de patrimonio escultórico & 4,00 & 3,91 \\
\hline Una impresora 3D en el aula es un buen recurso para la formación & 4,00 & 4,28 \\
\hline
\end{tabular}

Tabla 2. Resultados de la encuesta sobre uso de réplicas en educación. 


\section{Conclusiones y futuros trabajos}

Con esta experiencia se han explorado varios aspectos importantes sobre las réplicas de patrimonio escultórico:

- Existen programas gratuitos, que necesitan una instrucción mínima para generar modelos 3D digitales del patrimonio escultórico de una ciudad. Sólo se necesita tomar fotografías y el proceso de reconstrucción 3D tiene lugar automáticamente en la nube.

- Es posible crear una réplica de una escultura a un coste que oscila entorno a 1 euro si disponemos de una impresora propia o de un coste entre 23 y 90 euros en materiales plásticos si no disponemos de una impresora propia. Estas cuantías, dependiendo del material y el número de réplicas a realizar, nos permitirían decidir cuando un centro escolar debe comprar su propia impresora y cuando debe utilizar servicios de impresión.

- Se pueden crear réplicas en diferentes materiales utilizando los servicios de impresión online. Los precios aumentan considerablemente si se decide utilizar materiales como acero inoxidable, bronce u otros.

- Tanto los profesores como los alumnos consideran que las réplicas de esculturas pueden ayudar a su proceso de formación $(4,25$ y 4,00 sobre 5,00). Por otro lado, les gustaría tener un repertorio de réplicas de esculturas para utilizarlas en educación $(4,00$ y 3,74 sobre 5,00$)$.

- La impresión en 3D es una tecnología muy bien valorada para su incorporación en el aula $(4,00$ y 4,28 sobre 5,00$)$.

Por lo tanto, ni el coste, ni la dificultad técnica, suponen un impedimento para utilizar estas réplicas en entornos educativos y los posibles usuarios de las mismas demuestran un evidente interés en su utilización.

Se pretende como futuros trabajos, diseñar materiales y actividades docentes que permitan introducir el uso de réplicas de patrimonio escultórico en entornos educativos utilizando las tecnologías descritas en este artículo.

\section{Referencias}

Almagro, M. J. (1988). La utilidad de sustitutos y repreduciones en los Museos. Boletín de la Anabad, 38 (3), 177-186.

Arribas, A., Bermúdez, E., Blanco, S., Durán, J., Garrido, G., Gumiel, J., et al. (2004). Nuevos registros paleontológicos de grandes mamíferos en la Cuenca de GuadixBaza (Granada): aportaciones del Proyecto Fonelas al conocimiento sobre las faunas continentales del Plioceno-Pleistoceno europeo. Boletín Geológico y Minero, 115 (3), 567-582.

Boletín Oficial del Estado. (2006, 7-Julio). BOE $n^{\circ}$ 162. Retrieved 2014, 1-Julio from: http://www.boe.es/boe/dias/2006/07/08/pdfs/A25561-25572.pdf

Boletín Oficial de Canarias. (2007, 12-Junio). BOC - Decreto 158/2007, de 12 de Junio. Retrieved 2014, 1-Julio from www.gobiernodecanarias.org/boc: http://www. gobiernodecanarias.org/boc/2007/126/boc-2007-126-004.pdf

Bourke, P. (2012). Automatic 3D reconstruction: An exploration of the state of the art. The GSTF Journal on Computing;Oct., 2012, Vol. 2 Issue 3, p. 71, 2 (3), 71. 
British Geological Survey. (2013). GB3D Type Fossils. Retrieved 2014, 1-Julio from GB3D Type Fossils: http://www.3d-fossils.ac.uk/home.html

Brutto, M. L., \& Meli, P. (2012). Computer vision tools for 3D modelling in archaeology. International Journal of Heritage in the Digital Era, 1, 1-6.

Department for Education of United Kingdom. (2013). Report 3D printers in schools: uses in the curriculum. Enriching the teaching of STEM and design subjects. Department for Education (DfE) of United Kingdom.

Canessa, E., Fonda, C., \& Zennaro, M. (2013). Low-cost 3D Printing for Science, Education \& Sustainable Development. Trieste, Italy: ICTP.

Crasbom, J., \& Orrego, M. (2007). La elaboración de réplicas: Un instrumento de conservación y protección al patrimonio cultural. XX Simposio de Investigaciones Arqueológicas en Guatemala (pp. 187-199). J. P. Laporte, B. Arroyo y H. Mejía.

Czapka, J. T. (2002). Application of Rapid Prototyping Technology to Improve Spatial Visualization in an Introductory Engineering Graphics Course. University of Illinois at Urbana-Champaign.

Educastur. (2008, 5-Octubre). Restauras. Blog sobre recursos de Conservación y Restauración de Bienes Culturales. Retrieved 2014, 2-Julio from Reproducción de Esculturas: http://blog.educastur.es/restauras/2008/10/05/reproduccion-deesculturas/

Eurydice, E. (2009). Educación artística y cultural en el contexto escolar europeo. Bruselas: Secretaría General Técnica.

Gonizzi, S., Remondino, F., \& Visintini, D. (2012). Photogrammetry and Laser Scanning for Archaeological Site 3D Modeling - Some Critical Issues. Proceedings of the 2nd Workshop on The New Technologies for Aquileia. Aquileia, Italy.

Hernández, A. (2007). La clonación arquitectónica. Madrid: Siruela.

Johnson, W. M., Coates, C. W., Hager, P., \& Stevens, N. (2009). Employing Rapid Prototyping in a First-Year Engineering Graphics Course. 2009 ASEE Southeast Section Conference.

Kersten, T. P., \& Lindstaedt, M. (2012). Image-Based low-cost systems for automatic 3D recording and modelling of archaeological finds and objects. Proceeding EuroMed'12 Proceedings of the 4th international conference on Progress in Cultural Heritage Preservation (pp. 1-10). Berlin: Springer.

La Laguna Ahora. El periódico digital de La Laguna. (2009, 2-Agosto). TEA Tenerife Espacio de las Artes. Retrieved 2014, 2-Julio from La Laguna Ahora. El periódico digital de La Laguna: $\mathrm{http} / / / \mathrm{www}$. lalagunaahora.com/hemeroteca//content/view/9897/

Lerma, J. L., Cabrelles, M., \& Seguí, A. E. (2011). Aplicación de la fotogrametría terrestre al levantamiento de alzados de edificios singulares. Revista ph-Instituto Andaluz del Patrimonio Histórico (77), 127-129.

Luzón, J. M. (2003). Sobre la copia de antigüedades romanas y el caso del Westmorland. Actas de los XIII cursos monográficos sobre el patrimonio histórico. (pp. 17-30). Santander: Ed. Universidad de Cantabria.

MakerBot. (2008, Noviembre). David Renaissance sculpture by Michelangelo; Rodin's The Thinker. Retrieved 2014, 1-Julio from thingiverse: http://www.thingiverse.com/ thing:261508; http://www.thingiverse.com/thing:34343 
Manferdini, A., \& Galassi, M. (2013). Assessments for 3d reconstructions of cultural heritage using digital technologies. The International Archives of the Photogrammetry, Remote Sensing and Spatial Information Sciences, XL-5 (W1), 167-174.

Marcus Institute for Digital Education in the Arts. (2009). MIDEA. Retrieved 2014, 1-Julio, from: www.midea.nmc.org

Martín, N., de la Torre, J., \& Saorín, J. L. (2014). Prototipado Digital, Fabricación e Impresión 3D. Talleres Prácticos. bubok ed. ISBN: 978-84-686-5345-7.

Morris, R. (2014, 30-Marzo). Una aldea alpina o Venecia en miniatura: ¿por qué China copia ciudades enteras? (BBC, Producer) Retrieved 2014, 1-Julio, from BBC: http://www.bbc.co.uk/mundo/noticias/2014/03/140324_china_arquitectura replica_ciudades_finde_vp.shtml

MoveFab. (2013). MoveFab: Programa piloto de fomento de la creatividad y el talento a través de la fabricación digital. Retrieved 2014, 9-Julio from MoveFab: http:// www.fg.ull.es/movefab/

New Media Consortium. (2013). Horizon Report Edición sobre Educación Superior 2013. New Media Consortium. Austin: New Media Consortium.

Pomaska, G. (2013). Monitoring the deterioration of stone at Mindener Museum's Lapidarium. XXIV International CIPA Symposium SR3, XL-5/W2.

Scalfani, V., \& Sahib, J. (2013). A Model for Managing 3D Printing Services in Academic Libraries. (U. o. Andrea L. Duda, Ed.) Issues in Science and Technology Librarianship (72).

Schmidt, R., \& Ratto, M. (2013). Design Tools for the Rest of Us: Maker Hardware Requires Maker Software. Conference Proceedings: FAB at CHI Workshop.

Saorín, J. L., de la Torre, J., Zanardi, E., Martín, N., \& Carbonell, C. (2013). Transformación de diseños virtuales $3 D$ en maquetas reales mediante el uso de impresoras $3 D$ de bajo coste. Universidad de La Laguna, La Laguna, Tenerife.

Swedish ICT. (2014, 20-Febrero). Swedish ICT Interactive. Retrieved 2014, 8-Julio, from Groundbreaking visualization and 3D technologies reveal hidden ancient Egyptian treasures: https://www.tii.se/media/news/visualization-and-3d-technologies-revealhidden-treasures

Torres, J., Arroyo, G., Romo, C., \& De Haro, J. (2012). 3D Digitization using Structure from Motion. In I. N. (Editors) (Ed.), CEIG -Spanish Computer Graphics Conference.

Tourist Information Goslar. (2014). Goslar Ciudad Imperial. Retrieved 2014, 1-Julio, from http://www.goslar.de/kultur-freizeit/kunst-kaiserring

Troxler, P., \& Wolf, P. (2010). Bending the Rules. The Fab Lab Innovation Ecology. 11 International CINet Conference. Zurich, Switzerland.

Tsioukas, V. (2013). Free software solutions for the creation and manipulation of 3D representations of historical maps. e-Perimetron, 8 (3), 56-59.

UNESCO. (2006). Textos Básicos de la Convención del Patrimonio Mundial 1972. Textos Básicos de la Convención del Patrimonio Mundial 1972 (pág. 245). Francia: UNESCO.

Wayne M. Johnson, C. W. (2009). Employing Rapid Prototyping in a First-Year Engineering Graphics Course. ASEE Southeast Section Conference. Marietta, Georgia: Southern Polytechnic State University Marietta, Georgia.

Weinberg, M. (2013). What's the deal with copyright and 3D printing?. Institute for Emerging Innovation (IEI). Institute for Emerging Innovation (IEI). 